\title{
REVIEW ON APPLICATION AND FACTOR AFFECTING AND OFFICIAL MONOGRAPHS IN DISSOLUTION PROCESS
}

\author{
Mohammad Zishan*¹, Mohammad Amir ${ }^{1}$, Zeeshan Ahmad ${ }^{1}$, Mohd Wasim Hussain ${ }^{1}$, \\ Prashant Singh ${ }^{2}$, Sahar Idris ${ }^{2}$ \\ ${ }^{1}$ Department of Pharmaceutics, Integral University Kursi Road Lucknow, India \\ ${ }^{2}$ Department of Pharmacology, Integral University Kursi Road Lucknow, India
}

Article Info: Received 16 March 2017; Review Completed 28 April 2017; Accepted 28 April 2017, Available online 15 May 2017 Cite this article as:

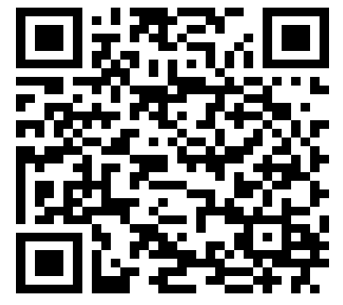

Zishan M, Amir M, Ahmad Z, Hussain MW, Singh P, Idris S, Review on application and factor affecting and official monographs in dissolution process , Journal of Drug Delivery and Therapeutics. 2017; 7(3):19-27

DOI: http://dx.doi.org/10.22270/jddt.v7i3.1422

*Address for Correspondence

Mohammad Zishan, Department of Pharmaceutics, Integral University, Dasauli, Kursi Road Lucknow, 226026, E-mail: zishanquadri786@gmail.com

\begin{abstract}
Dissolution testing is a critical methodology which is widely utilized in the development of a new pharmaceutical product. The test, in its simplest form, consists of placing the formulation in a dissolution apparatus containing suitable dissolution medium, allowing it to dissolve over a specified period of time and then assaying the resultant solution using appropriate analytical method to determine the amount of drug. Dissolution tests are relevant for an array of investigations like drug degradation profiles, stability and shelf life studies, physical and mechanical testing of dosage forms, incoming QC testing on raw materials etc. The present review outlines the recent findings on various dissolution apparatuses, their modifications, methods for degassing of media like Helium sparging, Heating and filtering, Vacuum degassing, sonication and dissolution testing of various dosage forms like Immediate Release (IR) Dosage forms, Delayed Release Dosage Forms, Extended Release Dosage Forms, Transdermal Delivery Systems, Powders, Chewable Tablets, Buccal Tablets, Chewing Gums, Soft Gelatin Capsule, Aerosols, Suppositories and other Semisolids.
\end{abstract}

Keywords: Dissolution, Factor affecting dissolution, Dissolution apparatus

\section{INTRODUCTION}

Most medicinal drugs are formulated into tablets, capsules or other forms of medicine. Formulating a medicine means mixing the medicinal drug with other ingredients (called excipients) according to a prescribed recipe (the formulation). These ingredients have a number of purposes in a tablet, for example, they might help bind the tablet together, control the rate of release of the drug and improve the taste of the tablet. Dissolution of a tablet involves its disintegration into smaller and smaller particles from which the medicinal drug is released more and more rapidly. The speed at which a medicinal drug is released from a tablet or capsule and dissolves in solutions that mimic fluids in the GI tract is an increasingly important measurement. Knowledge of this rate of dissolution contributes to the formulation, development and regulatory approval of medicines. ${ }^{1}$ It is also important for quality control, checking that the tablets from a production run have the required characteristics. The process of dissolution followed by absorption determines, in part, the bioavailability of the drug. The rate of dissolution can be determined in vivo by taking samples of a person's plasma or urine and measuring the drug concentration in them. However, this is not appropriate for routine measurements on the vast numbers of compounds investigated during drug discovery and development. 
Instead, in vitro tests are used. Fluids in the body are simulated and dissolution experiments carried out in laboratory glassware. Conditions for these tests are carefully defined. ${ }^{1,2,3,4,5}$

Dissolution of a tablet depends on ${ }^{2}$

$>$ The size of the granules of medicinal drug;

$>$ The structure of the tablets and the nature of the excipients used in the formulation;

$>$ The $\mathrm{pH}$ of fluids in the GI tract.

$>$ It also depends on temperature, but since body temperature is always $37{ }^{\circ} \mathrm{C}$ (or very close) temperature is not a factor in this situation.

\section{Importance of dissolution $1,2,5$}

Results from in-vitro dissolution rate experiments can be used to explain the observed differences in in-vivo availability.

$>$ Dissolution testing provides the means to evaluate critical parameters such as adequate bioavailability and provides information necessary to formulator in development of more efficacious and therapeutically optimal dosage forms.

$>$ Most sensitive and reliable predictors of in-vivo availability.

$>$ Dissolution analysis of pharmaceutical dosage forms has emerged as single most important test that will ensure quality of product.

$>$ It can ensure bioavailability of product between batches that meet dissolution criteria.

$>$ Ensure batch-to-batch quality equivalence both invitro and in-vivo, but also to screen formulations during product development to arrive at optimally effective products.

$>$ Physicochemical properties of model can be understood needed to mimic in-vivo environment.

$>$ Such models can be used to screen potential drug and their associated formulations for dissolution and absorption characteristics.

$>$ Serve as quality control procedures, once the form of drug and its formulation have been finalized.

\section{Application of Dissolution}

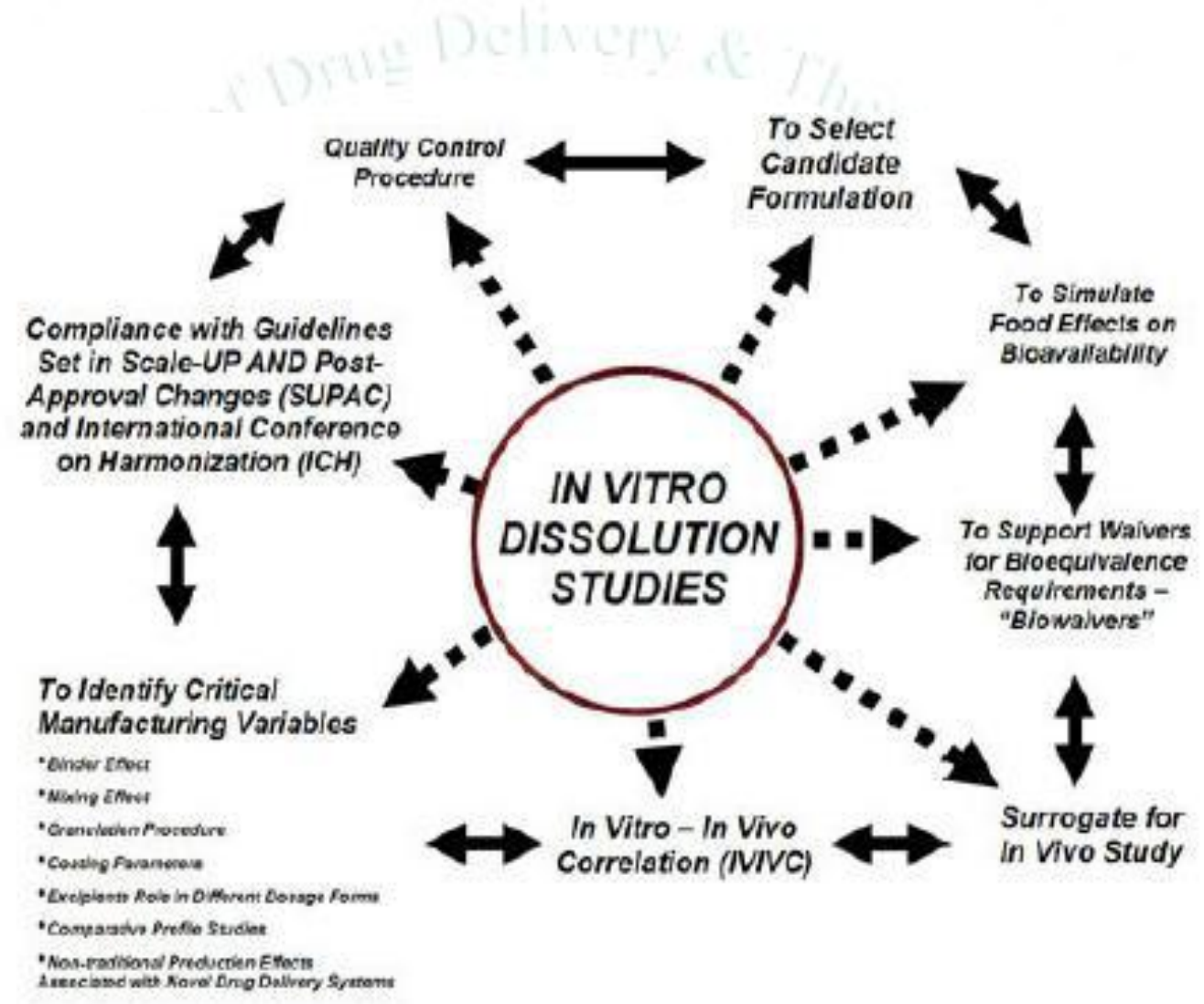

Figure 1: application of dissolution

\subsection{Product development ${ }^{1,2,5,7,8}$}

$>$ Important tool during development of dosage form.

$>$ Aids in guiding the selection of prototype formulations and for determining optimum levels of ingredients to achieve drug release profiles, particularly for extended release formulations.

$>$ Also guides in selection of a "market-image" product to be used in pivotal in-vivo bioavailability or bioequivalence studies.

\subsection{Quality assurance ${ }^{8}$}

D.T. performed on future production lots and is used to assess the lot-to-lot performance characteristics of drug product and provide continued assurance of product integrity/similarity.

\subsection{Product stability}

In-vitro dissolution also used to assess drug product quality with respect to stability and shelf-life. As product 
age, physicochemical changes to the dosage form may alter dissolution characteristics of drug product over time. For some products, polymorph transformations to more stable, and hence less soluble crystalline forms may result in reduced dissolution rates. ${ }^{8}$

\subsection{Comparability assessment}

Also useful for assessing the impact of pre- or postapproval changes to drug product such as changes to formulation or manufacturing process. Thus, in-vitro comparability assessment is critical to ensure continued performance equivalency and product similarity. ${ }^{8}$

\subsection{Waivers of in-vivo bioequivalence requirements}

In-vitro dissolution testing or drug release testing may be used for seeking waiver of required product to conduct in-vivo bioavailability or bioequivalence studies. ${ }^{8}$

\section{Factor affecting dissolution rate ${ }^{1.7,8}$ \\ $>$ Physicochemical Properties of Drug \\ $>$ Drug Product Formulation Factors \\ $>$ Processing Factors \\ $>$ Factors Relating Dissolution Apparatus \\ $>$ Factors Relating Dissolution Test Parameters}

\subsection{Physicochemical properties of drug ${ }^{2,8,9}$}

\subsubsection{Drug solubility}

Solubility of drug plays a prime role in controlling its dissolution from dosage form. Aqueous solubility of drug is a major factor that determines its dissolution rate. Minimum aqueous solubility of $1 \%$ is required to avoid potential solubility limited absorption problems. Studies of 45 compound of different chemical classes and a wide range of solubility revealed that initial dissolution rate of these substances is directly proportional to their respective solubility. ${ }^{2}$

\subsubsection{Salt formation}

It is one of the common approaches used to increase drug solubility and dissolution rate. It has always been assumed that sodium salts dissolve faster than their corresponding insoluble acids. Eg. sodium and potassium salts of Peniciilin G, sulfa drugs, phenytoin, barbiturates etc. While in case of Phenobarbital dissolution of sodium salt was slower than that of weak acid. Same is the case for weak base drug, strong acid salts, such as hydrochlorides and sulphates of weak bases such as epinephrine, tetracycline are commonly used due to high solubility. However, free bases of chlortetracycline, methacycline were more soluble than corresponding hydrochloride salt at gastric $\mathrm{pH}$ values, due to common ion suppression. ${ }^{7}$

\subsubsection{Particle size}

There is a direct relationship between surface area of drug and its dissolution rate. Since, surface area increases with decrease in particle size, higher dissolution rates may be achieved through reduction of particle size. Micronization of sparingly soluble drug to reduce particle size is by no means a guarantee of better dissolution and bioavailability. Micronization of hydrophobic powders can lead to aggregation and floatation when powder is dispersed into dissolution medium. So, mere increase in S.A. of drug does not always guarantee an equivalent increase in dissolution rate. Rather, it is increase in the "effective" S.A., or area exposed to dissolution medium and not the absolute S.A. that is directly proportional to dissolution rate.Hydrophobic drugs like phenacetin, aspirin shows decrease in dissolution rate as they tend to adsorb air at the surface and inhibit their wettability. Problem eliminated by evacuating surface from adsorbed air or by use of surfactants. So these drugs in-vivo exhibit excellent wetting due to presence of natural surfactants such as bile salts. $1,2,8$

\subsubsection{Solid state characteristics}

Solid phase characteristics of drug, such as amorphicity, crystallinity, state of hydration and polymorphic structures have significant influence on dissolution rate. Anhydrous forms dissolve faster than hydrated form bcz they are thermodynamically more active than hydrates. Eg. Ampicillin anhydrate faster dissolution rate than trihydrate. Amorphous forms of drug tend to dissolve faster than crystalline materials. E.g. Novobiocin suspension, Griseofulvin. ${ }^{9,10}$

\subsubsection{Co-precipitation}

Dissolution rate of sulfathiazole could be significantly increased by co-precipitating the drug with povidone. ${ }^{8}$

\subsection{Drug Product Formulation Factors}

Dissolution rate of pure drug can be altered significantly when mixed with various adjuncts during manufacturing process such as diluents, dyes, binders, granulating agents, disintegrants and lubricants. Generically identical tablet or capsules exhibited differences in their dissolution rates of their active ingredients. ${ }^{5,10}$

\subsubsection{Diluents}

Diluents in capsule \& tablet influence the dissolution rate of drug. Studies of starch on dissolution rate of salicylic acid tablet by dry double compression process shows three times increase in dissolution rate when the starch content increases from the 5-20\%. Here starch particles form a layer on the outer surface of hydrophobic drug particles resulting in imparting hydrophilic character to granules \& thus increase in effective surface area $\&$ rate of dissolution. Different types of dissolution apparatus utilized affect ranking of different varieties of starch. With stirring type of agitation, order was potato starch > cornstarch > arrowroot starch $>$ rice starch. With oscillating type, a different order observed. Corn $>$ rice $>$ arrowroot $>$ potato.The dissolution rate is not only affected by nature of the diluent but also affected by excipient dilution ${ }^{2}$

\subsubsection{Disintegrants}

Disintegrating agent added before \& after the granulation affects the dissolution rate. Studies of various disintegrating agents on Phenobarbital tablet showed that when copagel (low viscosity grade of $\mathrm{Na}$ CMC) added before granulation decreased dissolution rate but if added after did not had any effect on dissolution rate. Microcrystalline cellulose is a very 
good disintegrating agent but at high compression force, it may retard drug dissolution. Starch is not only an excellent diluent but also superior disintegrant due to its hydrophilicity and swelling property. Disintegration and dissolution rate of disintegrants with moderate swelling capacity depend to a large extent on mixing time of drug/excipient preblende. With lubricant. On other hand, disintegrants with strong swelling capacity such as sodium starch glycolate were hardly affected by mixing time with lubricant. ${ }^{2,10,11,12,13}$

\subsubsection{Binders and Granulating agents}

The hydrophilic binder increase dissolution rate of poorly wet table drug. Large amt. of binder increase hardness \& decrease disintegration /dissolution rate of tablet. Non aqueous binders such as ethyl cellulose also retard the drug dissolution Phenobarbital tablet granulated with gelatin solution provide a faster dissolution rate in human gastric juice than those prepared using NaCMC or polyethylene glycol 6000 as binder. Gelatin imparted hydrophilic character to hydrophobic drug surface whereas PEG 6000 formed a poorly soluble complex while NA-CMC was converted to its less soluble acid form at the low $\mathrm{pH}$ of gastric fluid. ${ }^{2,8}$

\subsubsection{Lubricants}

Lubricants are hydrophobic in nature (metallic stearates) and prolong tablet disintegration time by forming water repellant coat around individual granules. This retarding effect is most imp factor in influencing rate of dissolution of solid dosage forms. Both amount and method of addition affect the property. It should be added in small amount ( $1 \%$ or less) and should be tumbled or mixed gently for only very short time. Prolonged mixing the dissolution time. However, if an enhancing effect in dissolution of hydrophobic granules is desired, water soluble lubricant such as SLS or CARBOWAXES may be used. ${ }^{8}$

\subsubsection{Surfactants}

They enhance the dissolution rate of poorly soluble drug. This is due to lowering of interfacial tension, increasing effective surface area, which in turn results in faster dissolution rate. E.g Non-ionic surfactant Polysorbate 80 increase dissolution rate of phenacetin granules. The increase was more pronounced when the surfactant was sprayed on granules than when it was dissolved in granulating agent.

\subsubsection{Water-soluble dyes}

Dissolution rate of single crystal of sulphathiazole was found to decrease significantly in presence of FD\&C Blue No.1. The inhibiting effect was related to preferential adsorption of dye molecules on primary dissolution sources of crystal surfaces. They inhibit the micellar solubilization effect of bile salts on drug. Cationic dyes are more reactive in lower conc. than are anionic dyes. ${ }^{3}$

\subsubsection{Coating polymers-}

Tablets with MC coating were found to exhibit lower dissoln profiles than those coated with HPMC at $37^{\circ} \mathrm{C}$. The differences are attributed to thermal gelation of $\mathrm{MC}$ at temp near $37^{\circ}$, which creates a barrier to dissoln process \& essentially changes the dissoln medium. This mechanism is substantiated by the fact that at temp below the gel point \& at increased agitation, the effect disappears. 10

\subsection{Factors relating dissolution apparatus}

\subsubsection{Agitation}

Relationship between intensity of agitation and rate of dissolution varies considerably acc. to type of agitation used, the degree of laminar and turbulent flow in system, the shape and design of stirrer and physicochemical properties of solid. Speed of agitation generates a flow that continuously changes the liq/solid interface between solvent and drug. In order to prevent turbulence and sustain a reproducible laminar flow, which is essential for obtaining reliable results, agitation should be maintained at a relatively low rate. Thus, in general relatively low agitation should be applied. ${ }^{13}$

\section{Basket method- 100 rpm}

\section{Paddle method- 50-75 rpm}

\subsection{Factors relating dissolution test parameters}

\section{Temperature}

Drug solubility is temperature dependent, therefore careful temperature control during dissolution process is extremely important. Generally, a temp of $37^{\circ} \pm 0.5$ is maintained during dissolution determination of oral dosage forms and suppositories. However, for topical preparations temp as low as $30^{\circ}$ and $25^{\circ}$ have been used

\subsection{Dissolution medium}

It is very imp factor affecting dissolution and is itself affected by number of factors such as:

$>\mathrm{pH}$

$>$ Volume of dissolution medium

\section{Official dissolution monographs}

According to I.P. \& E.P. USP for solid dosage forms (tablets and capsules) dissolution apparatus used are:

$>$ Apparatus I-BASKET APPARATUS

$>$ Apparatus II - BASKET APPARATUS

According to B.P. apparatus used are:

$>$ Apparatus I - BASKET APPARATUS

$>$ Apparatus II - PADDLE APPARATUS

$>$ Apparatus III - FLOW THROUGH CELL APPARATUS 
Appratus-1

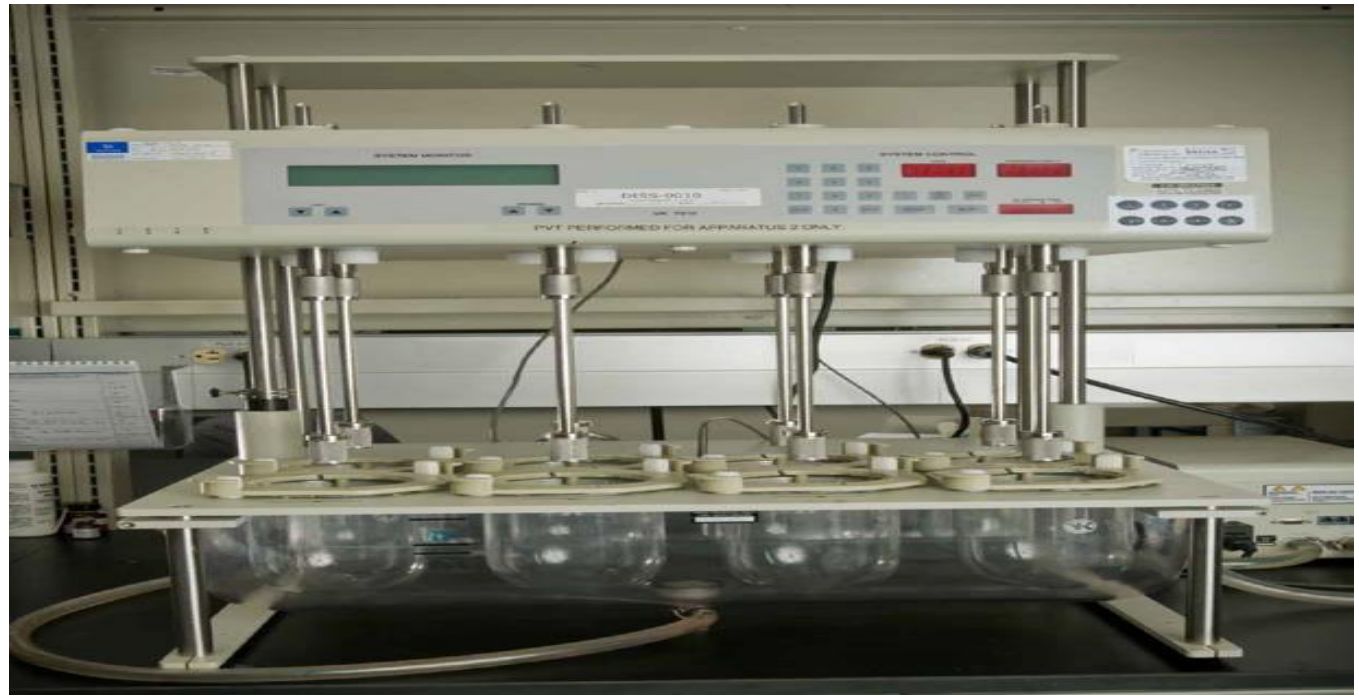

Figure 1: Appratus. 1

1. Temp. - $37 \pm 0.5 \circ \mathrm{C}$

2. $\mathrm{PH}- \pm 0.05$ unit in specified monograph

3. Capacity - $1000 \mathrm{ml}$

4. Distance between inside bottom of vessel and paddle/basket is maintained at $25 \pm 2 \mathrm{~mm}$.

5. For enteric coated dosage form it is first dissolved in $0.1 \mathrm{~N} \mathrm{HCl} \&$ then in buffer of $\mathrm{pH} 6.8$ to measure drug release. (Limit - NMT $10 \%$ of drug should dissolve in the acid after $2 \mathrm{hr}$.and about $75 \%$ of it should dissolve in the buffer after $45 \min ^{8}$

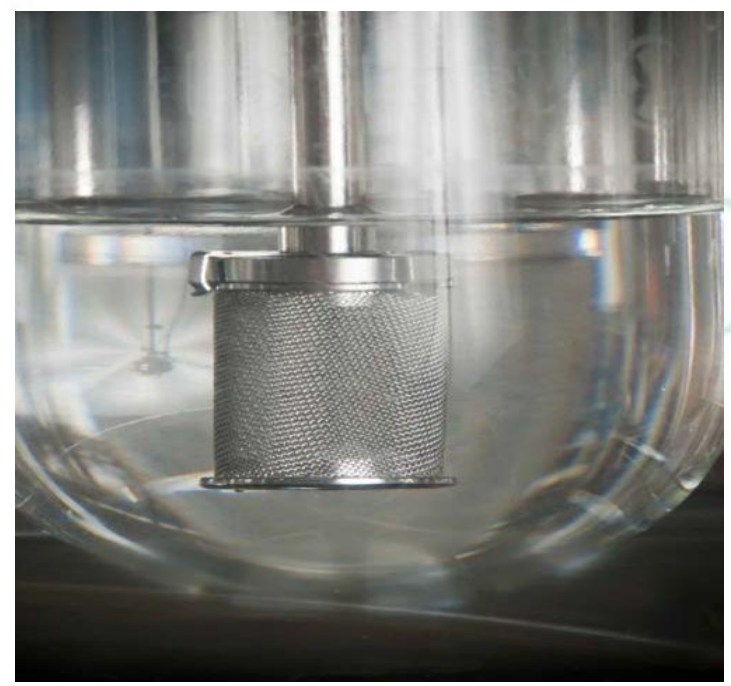

Figure 2: Type 1 Basket

\section{Advantage-}

\section{Drug products tested}

$>$ Solid dosage

$>$ Forms Floating

$>$ Disintegrating and non-disintegratingSingle units (e.g. tablets)

$>$ Multiple units (encapsulated beads)

$>\mathrm{pH}$ change by media addition or replacement.

\section{Disadvantages}

$>$ Formulation may clog the screen

$>$ Small disintegrated particles fall out

2) Apparatus-II - Paddle Apparatus. 2,8,10

$>$ The dosage unit is allowed to sink to the bottom of the vessel before rotation of the blade is started.

A small, loose piece of no reactive material such as not more than a few turns of wire helix may be attached to dosage units that would otherwise float.

Other validated sinker devices may be used.
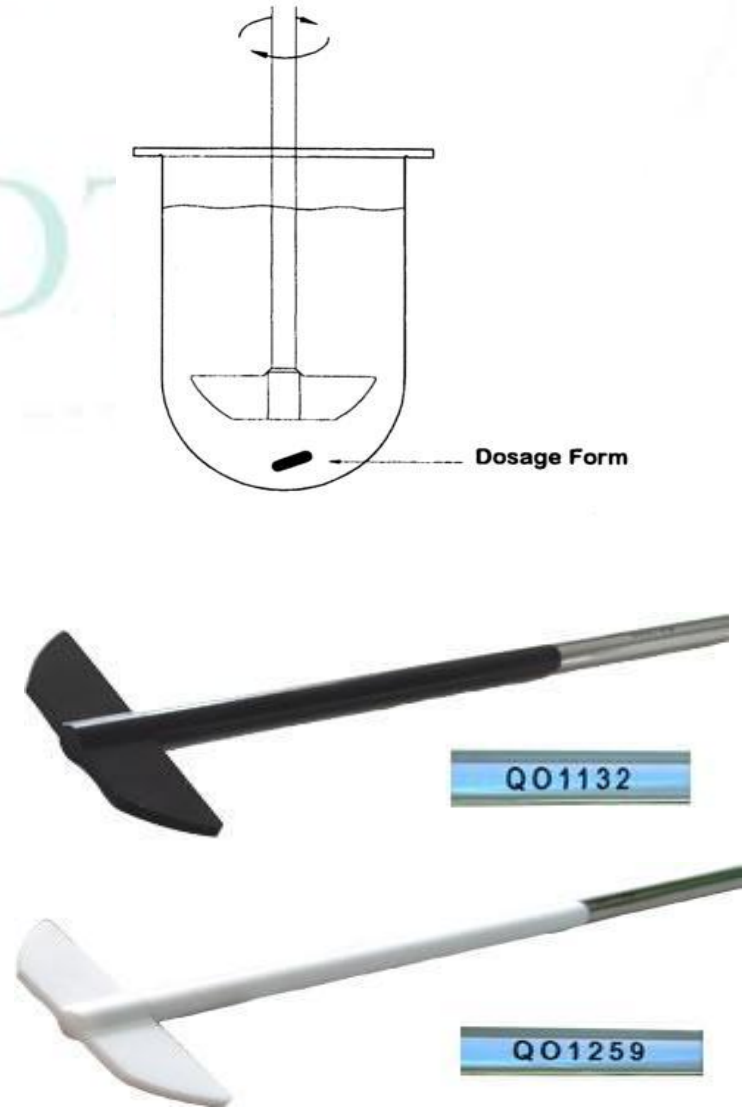

Figure 3: Apparatus. ii. 
Useful for: Tablets, Capsules, Beads, Delayed release, enteric coated dosage forms Standard volume: 900/1000 ml Advantages

$>$ Easy to use

$>$ Robust

$>$ Can be easily adapted to apparatus 5

$>$ long experience

$>\mathrm{pH}$ change possible

$>$ Can be easily automated which is important for routine investigations.

\section{Disadvantages}

$>\mathrm{pH} /$ media change is often difficult

$>$ Hydrodynamics are complex, they vary with site of the dosage form in the vessel (sticking, floating) and therefore may significantly affect drug dissolution

$>$ Coning.
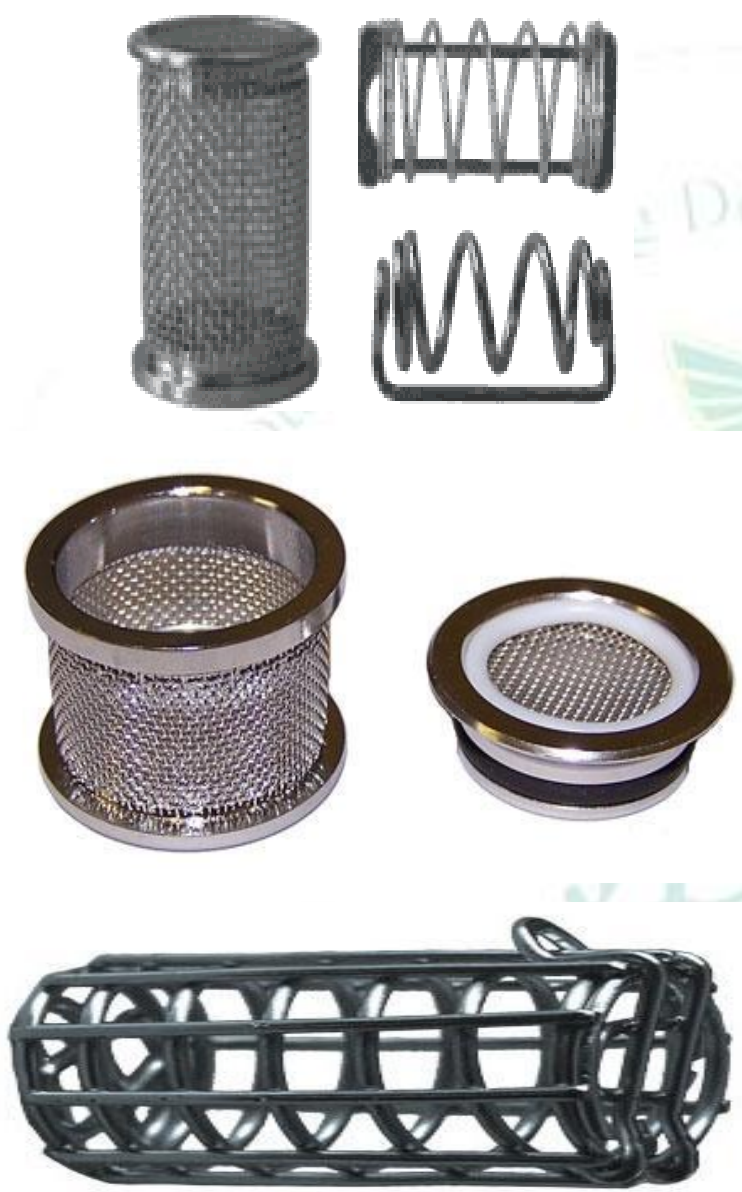

Figure 4: Sinkers for floating dosage forms

\section{Limitations of USP Apparatus 1and $2{ }^{9}$}

$>$ USP2 (and USP1) Apparatus has plenty of HYDRODYNAMICS.

$>$ Complicated 3-dimensional flow generated by the paddle.

$>$ Significant impact of convective transport Conditions used $(50-100 \mathrm{rpm})$ highly exaggerates flow in the GI.

$>$ If Static-tank model used - sink conditions artificially generated to simulate sink in GI.

$>$ Use of solvents and surfactants non-native to GI.
3) Apparatus III - Reciprocating cylinder 2,7,8,9,10

he assembly consists of a set of cylindrical, flatbottomed glass vessels; a set of glass reciprocating cylinders; stainless steel fittings (type 316 or equivalent) and screens that are made of suitable nonsorbing and nonreactive material(polypropelene) and that are designed to fit the tops and bottoms of the reciprocating cylinders; and a motor and drive assembly to reciprocate the cylinders vertically inside the vessels. The vessels are partially immersed in a suitable water bath of any convenient size that permits holding the temperature at $37 \pm 0.5$ during the test. The dosage unit is placed in reciprocating cylinder $\&$ the cylinder is allowed to move in upward and downward direction constantly. Release of drug into solvent within the cylinder measured.

Useful for: Tablets, Beads, controlled release formulations

Standard volume: $200-250 \mathrm{ml} /$ station

\section{Advantages:}

$>$ Easy to change the $\mathrm{pH}$-profiles

$>$ Hydrodynamics can be directly influenced by varying the dip rate.

\section{Disadvantages:}

$>$ small volume (max. $250 \mathrm{ml}$ )

$>$ Little experience

$>$ Limited data
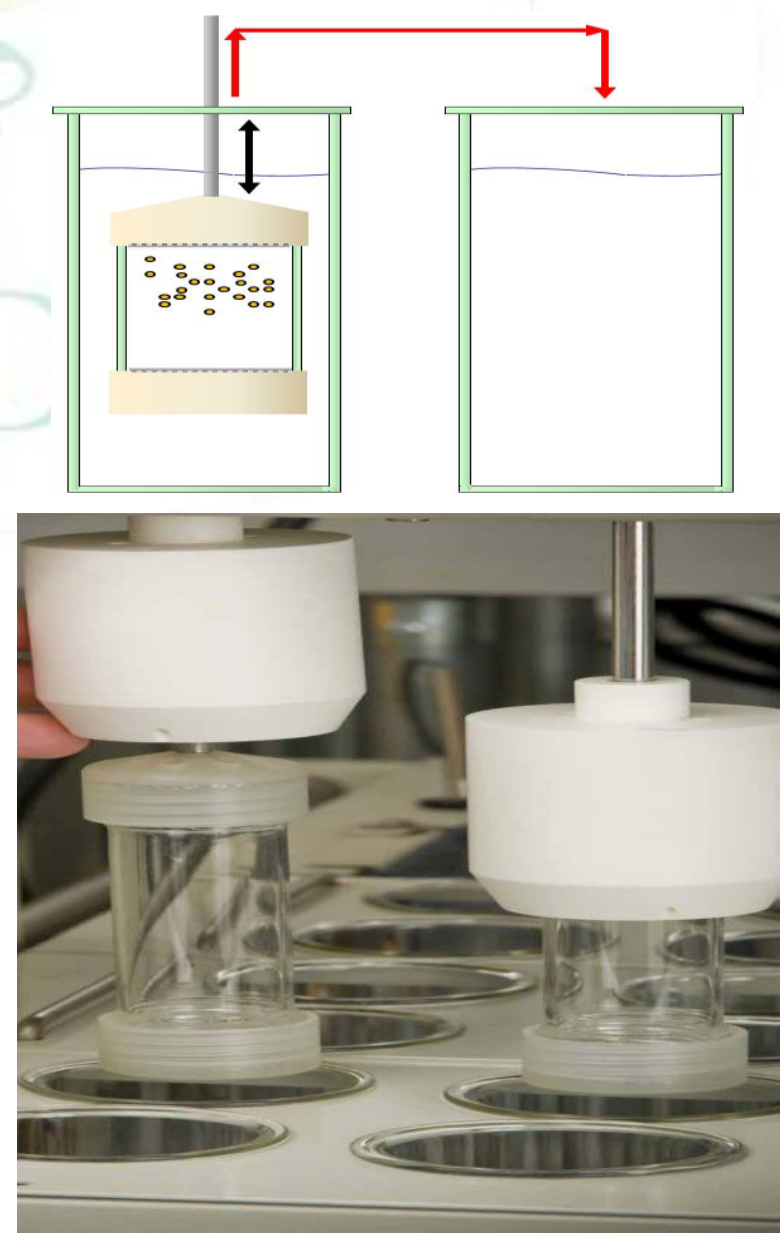

Figure 5: Apparatus iii 
4) Apparatus IV - flow through cell ${ }^{811}$

$>$ The assembly consists of a reservoir and a pump for the Dissolution Medium; a flow-through cell; a water bath that maintains the Dissolution Medium at $37 \pm 0.5$

$>$ The cell size is specified in the individual monograph.

> The pump forces the Dissolution Medium upwards through the flow-through cell.

$>$ Place the glass beads into the cell specified in the monograph.
$>$ Place 1 dosage unit on top of the beads or, if specified in the monograph, on a wire carrier.

$>$ Assemble the filter head, and fix the parts together by means of a suitable clamping device.

$>$ Introduce by the pump the Dissolution Medium warmed to $37 \pm 0.5$ through the bottom of the cell to obtain the flow rate specified in the individual monograph.

$>$ Collect the elute by fractions at each of the times stated.

$>$ Perform the analysis as directed in the individual monograph.
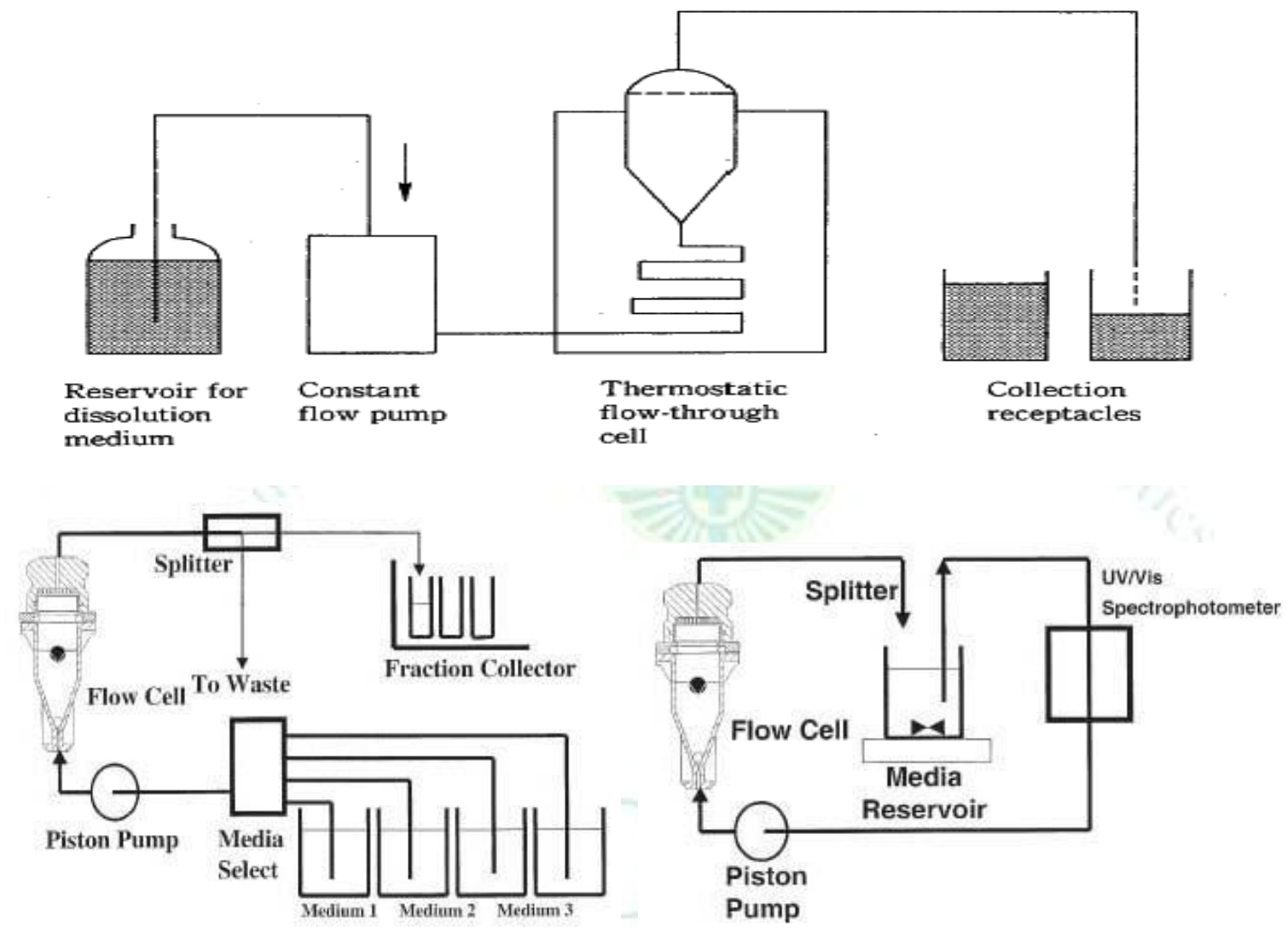

Figure 6: Apparatus iv

Useful for: Low solubility drugs, Micro particulates, Implants, Suppositories, Controlled release formulations

\section{Variations:}
(A) Open system
(B) Closed system

\section{Advantages:}

$>$ Easy to change media $\mathrm{pH}$

$>$ PH-profile possible

$>$ Sink conditions

\section{Disadvantages:}

$>$ Desecration necessary

$>$ High volumes of media

$>$ Labor intensive

5) Apparatus V - Paddle over disk ${ }^{2.5 .6 .8}$
Use the paddle and vessel assembly from Apparatus 2 with the addition of a stainless steel disk assembly designed for holding the transdermal system at the bottom of the vessel.

$>$ Other appropriate devices may be used, provided they do not sorb, react with, or interfere with the specimen being tested

$>$ The disk assembly for holding the transdermal system is designed to minimize any "dead" volume between the disk assembly and the bottom of the vessel.

$>$ The disk assembly holds the system flat and is positioned such that the release surface is parallel with the bottom of the paddle blade

$>$ The vessel may be covered during the test to minimize evaporation.

Useful for: Transdermal patches Standard volume: 900 $\mathrm{ml}$ 


\section{Disadvantages:}

Disk assemmbly restricts the patch size.
$>$ Borosilicate Glass

$>17$ mesh is standard (others available)

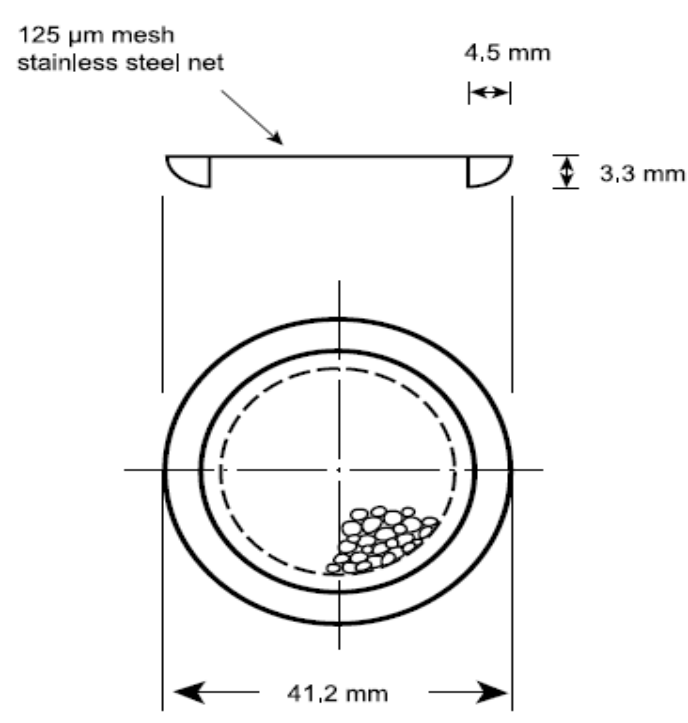

Figure 2.9.4.-1. - Disk assembly

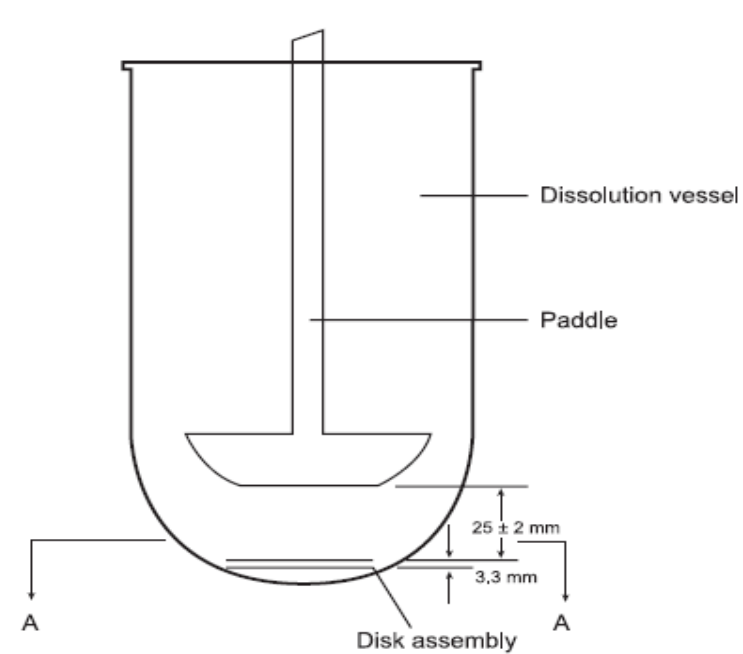

Figure 2.9.4.-2. - Paddle and disk

Figure 7: Apparatus V. Paddle over disk

\section{6) Apparatus VI - cylinder ${ }^{8}$}

$>$ Use the vessel assembly from Apparatus 1 except to replace the basket and shaft with a stainless steel cylinder stirring element and to maintain the temperature at $32 \pm 0.5$ during the test.

$>$ The dosage unit is placed on the cylinder at the beginning of each test, to the exterior of the cylinder such that the long axis of the system fits around the circumference of the cylinder $\&$ removes trapped air bubbles.

$>$ Place the cylinder in the apparatus, and immediately rotate at the rate specified in the individual monograph.

7) Apparatus VII - reciprocating holder ${ }^{8}$

$>$ The assembly consists of a set of volumetrically calibrated solution containers made of glass or other suitable inert material, a motor and drive assembly to reciprocate the system vertically and a set of suitable sample holders.

$>$ The solution containers are partially immersed in a suitable water bath of any convenient size that permits maintaining the temperature, inside the containers at $32 \pm 0.5$

$>$ For Coated tablet drug delivery system attach each system to be tested to a suitable sample holder (e.g., by gluing system edge with 2-cyano acrylate glue onto the end of a plastic rod or by placing the system into a small nylon net bag at the end of a plastic rod or within a metal coil attached to a metal rod).

$>$ For Transdermal drug delivery system attach the system to a suitable sized sample holder with a suitable O-ring such that the back of the system is adjacent to and centered on the bottom of the disk- shaped sample holder or centered around the circumference of the cylindrical-shaped sample holder. Trim the excess substrate with a sharp blade.

For Other drug delivery systems attach each system to be tested to a suitable holder as described in the individual monograph.

$>$ Suspend each sample holder from a vertically reciprocating shaker such that each system is continuously immersed in an accurately measured volume of Dissolution Medium within a calibrated container.

$>$ Reciprocate at a frequency of about 30 cycles per minute with amplitude of about $2 \mathrm{~cm}$, or as specified in the individual monograph, for the specified time in the medium specified for each time point.

$>$ Perform the analysis as directed in the individual monograph.

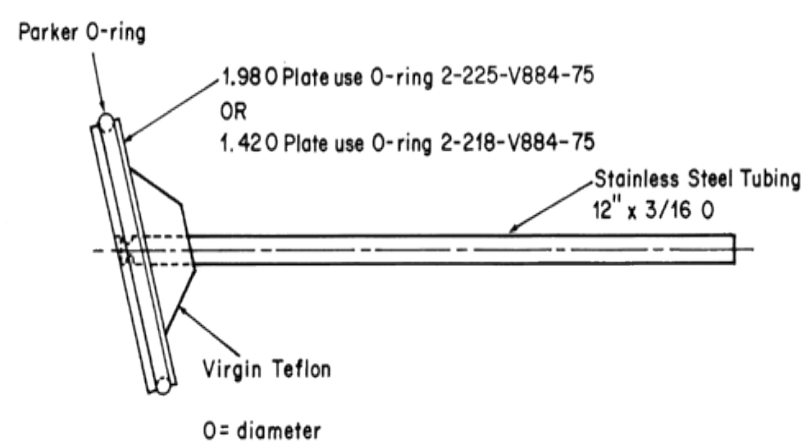

Figure 8: Apparatus VII - reciprocating holder 


\section{CONCLUSION}

At the conclusion of acceptable installation and operational qualifications, the dissolution apparatus is considered validated and acceptable for use to perform dissolution testing. The system suitability tests should be performed after any significant equipment change

\section{REFERENCES}

1. Brahmankar DM, Jaiswal SB. Biopharmaceutical and pharmacokinetics A treatise Vallaabh Prakashan, First edition, New Delhi 1995, pp 18-50.

2. Kushwaha P, Handbook of pharmaceuticak technology,first edition published by jaypee brothers Medical publishers (P) Ltd.2015,pp 60-72.

3. Gray VA, Brown CK, Dressman JB, Leeson LJ, A New General Information Chapter on Dissolution. Pharm. Forum 2001, 27 (6), 3432-3439.

4. Ross MSF, Rasis M, Mega Paddle,A Recommendation to Modify Apparatus Used in the USP General Test for Dissolution 711 . Pharm. Forum 1998, 24 (3), 6351-6359.

5. Howard C, Lloyd V, Allen J, Nicholas G, Popovich, Pharmaceutical Dosage Forms and Drug Delivery Systems. Baltimore,Maryland: Lippincott Williams \& Wilkins, 7th edition, 1999,

6. Reeta Rani Thakur, Sonia Narwal. Orally disintegrating preparations, recent advancement in formulation and technology. Journal of Drug Delivery \& Therapeutics, 2012, 2(3), 87-96.

7. Jayaprakash S, Mathew Ebin P Sovichan, Lalithcherian, Rani S, Praveen Raj R. Study on the effects of various disintegrants (e.g., a change from a basket apparatus to a paddle apparatus, unless multiple apparatus are qualified at the time of validation) or relocation of the dissolution apparatus (e.g., to another laboratory). Barring any significant change, the system suitability tests should be conducted at least twice a year as part of a robust preventive maintenance program. dispersible tablets. International Research Journal of Pharmacy, 2012, 3(5), 146-152.

8. United States Pharmacopeial Convention, Inc. United States Pharmacopeia 26. Rockville,Maryland: United States Pharmacopeial Convention, Inc. 2003.

9. Qureshi SA. The USP Dissolution Apparatus Suitability Test." Drug Information Journal. 1996; 30;1055-1061.

10. Desai D, Wang J, Wen H , Li X Timmins P. Formulation design, challenges, and development considerations for fixed dose combination (FDC) of oral solid dosage forms. Pharmaceutical Development and Technology, 2013, 18(6), 1265-1276.

11. Gupta AK, Arshad S, Poulter NR, Compliance, safety, and effectiveness of fixed-dose combinations of antihypertensive agents, a meta-analysis. Hypertension, 2010, 55(2), 399-407.

12. Akazawa M, Fukuoka K. Economic impact of switching to fixed-dose combination therapy for Japanese hypertensive patients, a retrospective cost analysis. a BMC Health Services Research, 2013, 124.

13. World Health Organization. WHO Expert Committee on Malaria (1998, Geneva, Switzerland). WHO expert committee on malaria, twentieth report. World Health Organization Technical Report Series, 2000, 892. 